\title{
Uterine Fistula, CTCAE
}

National Cancer Institute

\section{Source}

National Cancer Institute. Uterine Fistula, CT CAE. NCI Thesaurus. Code C143916.

A disorder characterized by an abnormal communication between the uterus and another organ or anatomic site. 